\title{
Fibroblast migration and collagen deposition during dermal wound healing: mathematical modelling and clinical implications
}

S. McDougall

\author{
J. A. Sherratt \\ P. K. Maini \\ J. C. Dallon \\ dallon@math.byu.edu
}

Follow this and additional works at: https://scholarsarchive.byu.edu/facpub

Part of the Mathematics Commons

\section{Original Publication Citation}

S. McDougall, J.C. Dallon, J. A. Sherratt, P. K. Maini: Fibroblast migration and collagen deposition during dermal wound healing: mathematical modelling and clinical implications. Phil. Trans. R. Lond. A 364:1385-145 (26)

\section{BYU ScholarsArchive Citation}

McDougall, S.; Sherratt, J. A.; Maini, P. K.; and Dallon, J. C., "Fibroblast migration and collagen deposition during dermal wound healing: mathematical modelling and clinical implications" (2006). Faculty Publications. 973.

https://scholarsarchive.byu.edu/facpub/973 


\title{
Fibroblast migration and collagen deposition during dermal wound healing: mathematical modelling and clinical implications
}

\author{
By Steven McDougall ${ }^{1}$, John Dallon ${ }^{2, *}$, Jonathan Sherratt ${ }^{3}$ \\ AND Philip Maini ${ }^{4}$ \\ ${ }^{1}$ Institute of Petroleum Engineering, and ${ }^{3}$ Department of Mathematics, \\ Heriot-Watt University, Edinburgh EH14 $4 A S, U K$ \\ ${ }^{2}$ Department of Mathematics, Brigham Young University, Provo, \\ UT 84602-6539, USA \\ ${ }^{4}$ Centre for Mathematical Biology, Mathematical Institute, 24-29 St Giles', \\ Oxford $O X 13 L B, U K$
}

The extent to which collagen alignment occurs during dermal wound healing determines the severity of scar tissue formation. We have modelled this using a multiscale approach, in which extracellular materials, for example collagen and fibrin, are modelled as continua, while fibroblasts are considered as discrete units. Within this model framework, we have explored the effects that different parameters have on the alignment process, and we have used the model to investigate how manipulation of transforming growth factor- $\beta$ levels can reduce scar tissue formation. We briefly review this body of work, then extend the modelling framework to investigate the role played by leucocyte signalling in wound repair. To this end, fibroblast migration and collagen deposition within both the wound region and healthy peripheral tissue are considered. Trajectories of individual fibroblasts are determined as they migrate towards the wound region under the combined influence of collagen/fibrin alignment and gradients in a paracrine chemoattractant produced by leucocytes. The effects of a number of different physiological and cellular parameters upon the collagen alignment and repair integrity are assessed. These parameters include fibroblast concentration, cellular speed, fibroblast sensitivity to chemoattractant concentration and chemoattractant diffusion coefficient. Our results show that chemoattractant gradients lead to increased collagen alignment at the interface between the wound and the healthy tissue. Results show that there is a trade-off between wound integrity and the degree of scarring. The former is found to be optimized under conditions of a large chemoattractant diffusion coefficient, while the latter can be minimized when repair takes place in the presence of a competitive inhibitor to chemoattractants.

Keywords: scar tissue; alignment; fibroblasts; chemoattractant; transforming growth factor- $\beta$

\footnotetext{
* Author for correspondence (dallon@math.byu.edu).

One contribution of 15 to a Theme Issue 'Biomathematical modelling II'.
} 


\section{Introduction}

In dermal wound healing, after the skin is injured, several interacting events are initiated including inflammation, tissue formation, angiogenesis, tissue contraction and tissue remodelling (Clark 1989). Crucial to all of these events is the interaction of the cells with the extracellular matrix (ECM). After the blood clot has formed during the inflammatory response, white blood cells invade the wound region by migrating through the ECM. Fibroblasts subsequently migrate into the region and begin to replace the blood clot with collagen. They biochemically alter the ECM by degrading the fibrin and producing collagen (Jennings \& Hunt 1992). While new tissue is being generated, endothelial cells migrate into the region forming a new vasculature in the process known as angiogenesis (Clark 1996). The optimal outcome is a healed wound with new tissue identical to that surrounding the wound but this is typically not the case. The new tissue is usually characterized by a new architecture that differs from the original and is frequently less functional than the original tissue. This remodelled tissue is known as a scar.

The characteristics of scars are a result of altered structure and composition in the dermis. Scars typically have fewer blood vessels supplying the denser connective tissue which is less elastic. The most significant difference between normal tissue and scar tissue seems to be the orientation of the fibrous matrix. In rodents, normal tissue has a reticular collagen pattern, whereas the collagen in scar tissue forms large parallel bundles at approximately right angles to the basement membrane (Whitby \& Ferguson 1991). Human scar tissue is similar, with greater collagen density, larger fibres and more alignment than normal tissue, although the alignment is parallel to the skin (Ehrlich \& Krummel 1996). Further structural differences between scars and normal tissue include a different ratio of collagen types and a lack of hair follicles and sweat glands. In the work discussed here we focus on the fibre alignment in scar tissue.

First we describe the wound healing process, then review our previous modelling work, and finally report new results. Our previous work will be divided into three parts. Our initial modelling of collagen alignment (Dallon et al. 1999) will be described in $\$ 3$, followed by a description of a model for wound repair (Dallon et al. 2000) in $\S 4$, and in $\S 5$, a model explaining the effects of transforming growth factor- $\beta$ (TGF $\beta$ ) (Dallon et al. 2001) will be reviewed. In $\S 6$, we begin reporting the new results by describing the extension of the model designed to examine the role of leucocyte signalling in wound repair, the role of fibroblast distribution ( $\$ 6 a)$ and the effect of the activity of a diffusible chemoattractant $(\$ 6 b)$. Finally, we end with a discussion in $\S 7$.

\section{Dermal wound healing}

There are fundamental differences between foetal and adult wound healing, resulting in different outcomes. In adults, the end product is a scar. However, foetal tissue is repaired with reduced or absent scarring (McCallion \& Ferguson 
1996) and the realization of this difference has generated a large volume of research activity into mechanisms underlying scar formation.

The healing of full-thickness skin wounds involves a complex sequence of overlapping events. One of the first is the coagulation cascade (Jennings \& Hunt 1992) where fibrinogen, a soluble protein in the plasma, is converted to fibrin which polymerizes to form a blood clot. This clot stops bleeding and can dry to form a scab which covers the wound; it also forms a provisional matrix which provides a scaffold for the invasion of various cell types. Within $24-48 \mathrm{~h}$ (Forrest 1983; Jennings \& Hunt 1992), fibroblasts start to infiltrate the wound and dissolve the fibrin clot replacing it with a collagen matrix. In the final phase of wound healing, the composition of the ECM is changed over a period of months, again by dermal fibroblasts.

Although several of the ECM-fibroblast interactions have been experimentally studied, this area remains poorly understood (Hay 1991). This is partly because all interactions have not been found, but mainly because the processes involved interact in a complex manner with nonlinear feedback. Mathematical modelling is a powerful tool designed to address such complex feedback mechanisms.

The following models assume a few well-known interactions which are briefly summarized. First, fibroblast movement is directed by the orientation of the matrix, a phenomenon known as 'contact guidance' (Hsieh \& Chen 1983; Clark et al. 1990; Guido \& Tranquillo 1993). Second, the ECM affects the speed of the fibroblasts. It is known that the matrix composition influences the motility of fibroblasts, which migrate more easily on fibronectin gels than on collagen gels (Wojciak-Stothard et al. 1997). Third, the composition of the ECM alters the production of different proteins by the fibroblasts (Clark et al. 1995; Xu \& Clark 1996). Fourth, the ECM in the wound region contains a plethora of growth factors and cytokines which alter fibroblast behaviour. And finally, fibroblasts organize the thin collagen fibrils into the fibrous structure seen in the dermis (Ehrlich \& Krummel 1996).

Two of these interactions are key to our models and deserve further explanation. Firstly, the phenomenon known as 'contact guidance' has been demonstrated directly when fibroblasts placed on oriented collagen gels invade the gels in the direction of orientation (Guido \& Tranquillo 1993). Further evidence of this behaviour is demonstrated when fibroblasts migrate along fibronectin fibrils (Hsieh \& Chen 1983). The second key interaction relates to the way in which collagen is produced by fibroblasts and the complex process by which the collagen polymerizes to form a fibrous network of matrix (Alberts et al. 2002). The fibroblasts release procollagen molecules via secretory vesicles. The fusion of these vesicles with the cell membrane creates deep, narrow recesses in the fibroblast's cell surface. It is in these recesses that the collagen fibrils are formed. It is theorized that these deep recesses give the fibroblast control over the micro-environment within which the collagen fibrils are forming and thus control over the structure of the resulting collagen matrix (Birk \& Trelstad 1986). The collagen matrix is itself an essential framework, which the migrating fibroblasts use as scaffolding to crawl along. Thus, not only do the fibroblasts affect the orientation of the matrix, but the matrix orientation also influences the directed movement of the fibroblasts. 


\section{The orientation model}

We began our modelling work with a multiscale model of a fibrous matrix and cells, which provides the foundation for our subsequent models. In this model we considered only one type of fibre, i.e. collagen, and one type of cell, fibroblasts. We represent the fibrous matrix as a continuum and the cells as discrete entities due to the scales involved. A moving fibroblast can range greatly in its length with typical values of the order $100 \mu \mathrm{m}$, and the diameters of the collagen fibres are of the order $1 \mu \mathrm{m}$ (Doillon et al. 1985; Birk \& Trelstad 1986). Additionally, normal dermal tissue is relatively sparsely populated with cells making a discrete representation more realistic, although during wound healing more cells are recruited to the region.

In the orientation model, cell paths are denoted by $\boldsymbol{f}^{i}(t)$ where the superscript denotes the particular cell. The fibrous matrix is denoted by $\boldsymbol{c}(\boldsymbol{x}, t)$ where $\boldsymbol{x}$ represents the Cartesian coordinates of a point in the plane. The vectors $\boldsymbol{c}$ have unit length and their direction indicates the predominant orientation of the fibrous matrix at the spatial location. The cells receive directional cues from the fibrous matrix (see equation (3.1)) and move in a direction determined by a weighted average of their previous direction of motion (persistence) and the direction of the fibrous matrix at their current location. The persistence term is incorporated into the formulation since fibroblasts tend to form and maintain a leading edge (Friedl et al. 1998).

In all the models, we assume that fibroblasts produce collagen aligned with their direction of movement. In this orientation model, we assume that production and degradation are balanced, so that the collagen density remains the same. However, the collagen orientation changes according to a weighted average of the velocities of nearby fibroblasts (equations (3.3) and (3.4)) as described in equation (3.5).

Thus, the equations for the model which govern the cell motion are:

$$
\frac{\mathrm{d} \boldsymbol{f}^{i}(t)}{\mathrm{d} t} \equiv \dot{\boldsymbol{f}}^{i}(t)=s \frac{\boldsymbol{v}^{i}(t)}{\left\|\boldsymbol{v}^{i}(t)\right\|}
$$

with

$$
\boldsymbol{v}^{i}(t)=(1-\rho) \boldsymbol{c}\left(\boldsymbol{f}^{i}(t), t\right)+\rho \frac{\dot{\boldsymbol{f}}^{i}(t-\tau)}{\left\|\dot{\boldsymbol{f}}^{i}(t-\tau)\right\|},
$$

where $\rho$ and $s$ are positive constants with $s$ representing the cell speed and $\tau$ a time lag. The equations which determine the fibrous matrix are:

$$
\boldsymbol{f}(\boldsymbol{x}, t)=\sum_{i=0}^{N} w_{i}(\boldsymbol{x}, t) \frac{\dot{\boldsymbol{f}}^{i}(t-\tau)}{\left\|\dot{\boldsymbol{f}}^{i}(t-\tau)\right\|},
$$

where $\tau$ is again a time lag and $N$ is the total number of cells. The weight function used here is defined by

$$
w_{i}(\boldsymbol{x}, t)=a_{1} a_{2} \quad \text { with } \quad a_{j}=\max \left\{1-\frac{\left|f^{i}(t)_{j}-x_{j}\right|}{L}, 0\right\},
$$


where $\boldsymbol{x}=\left(x_{1}, x_{2}\right)$ and $L=10 \mu \mathrm{m}$. Thus, the support of the function is a 20 by $20 \mu \mathrm{m}$ square. The square is used due to the lack of information in the model about the cell's shape. The change in the collagen orientation is described by

$$
\frac{\mathrm{d} \theta(\boldsymbol{x}, t)}{\mathrm{d} t}=\kappa\|\boldsymbol{f}\| \sin (\phi-\theta),
$$

where $\theta$ is the angle of $\boldsymbol{c}$, the vector representing the collagen direction and $\phi(\boldsymbol{x}, t)$ is the angle of $\boldsymbol{f}(\boldsymbol{x}, t)$. Thus, when the difference between the angles is small the derivative is small, and when the directions are orthogonal the rate of change reaches a maximum. In addition, it is periodic so that when a fibre and cell are oriented in either the same direction or $180^{\circ}$ apart, the fibre does not change direction.

The numerical algorithm consists of the following steps:

(i) Interpolate the direction of movement of the cell to the grid defining the fibres.

(ii) Orient the fibres with respect to the direction of the cell movement.

(iii) Interpolate fibre direction to the location of the cell.

(iv) Normalize the interpolated direction.

(v) Change the direction of the fibres and normalize.

(vi) Move the cell in the direction found in step (iii).

From this relatively simple model we can learn many things. Of the three parameters in the model $\rho, \kappa$ and $s$, the overall alignment of the matrix is least sensitive to $\rho$. In other words, the speed of the cells $s$ and the relative rate at which they modify the fibrous structure $\kappa$ seem to be more important in determining the overall alignment than the tendency of a cell to form and maintain a leading edge (figure 1).

In addition to changing the parameters of the model, the initial conditions can be varied. We examined variations in cell density, cell flux and the initial matrix orientation. Although increases in cell density do increase alignment, simulations lead us to conclude that cell density is less important in determining the overall alignment pattern than variations in the cell flux and the initial matrix orientation. A small region of alignment in the initial matrix can greatly influence the final overall alignment pattern. When cells enter the wound region from the same boundaries, the initial cells tend to form a region of alignment which the subsequent cells will reinforce and enhance.

\section{A model for dermal wound repair}

In dermal wound healing, fibroblasts move from normal tissue into a fibrin clot and over a period of time replace the fibrin matrix with a collagen-based matrix that forms the new tissue. We extend the orientation model to more closely mimic these processes, which are more complex than simple matrix orientation. This includes allowing for production and degradation of different ECM components by cells and some cellular control by the different matrix components (Dallon et al. 2000, 2001). 


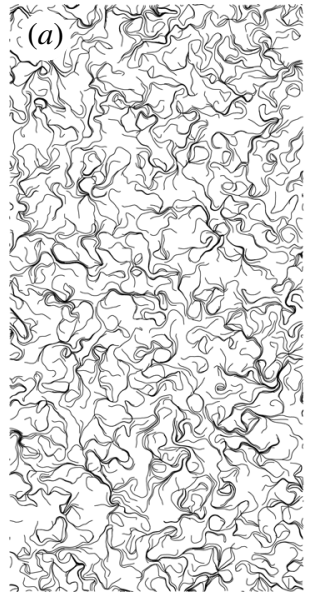

initial matrix

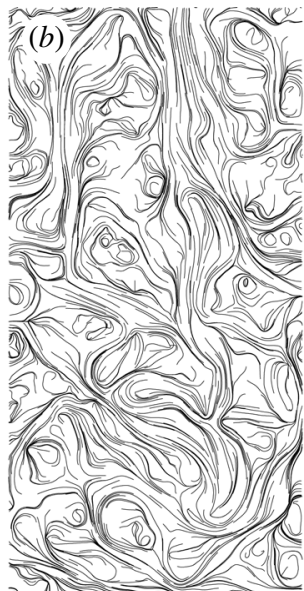

$\kappa=2.5$

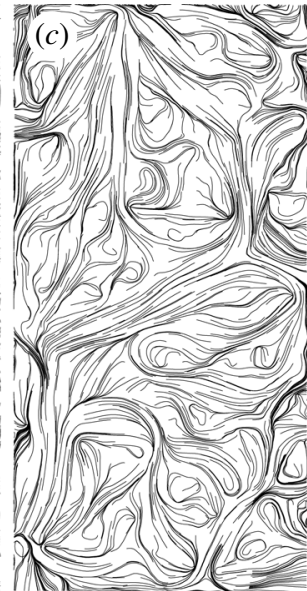

$\kappa=5$

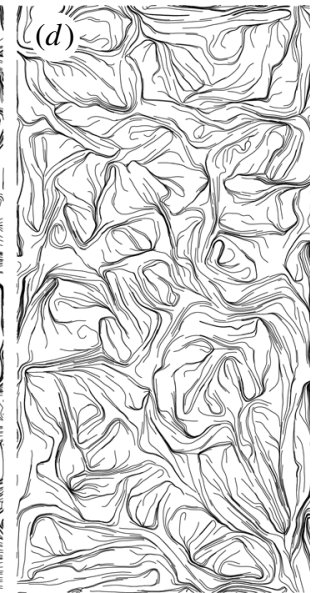

$\kappa=20$

Figure 1. The effect of altering the rate at which the fibroblasts change the fibre direction leads to a biphasic response of the structure inherent in the collagen orientation structure. That is, as $\kappa$ increases the orientation initially becomes less random and then with further increases it becomes more random. (a) Initial random fibrous matrix, $(b) \kappa=2.5,(c) \kappa=5$ and $(d) \kappa=20$. The collagen orientation is shown after $100 \mathrm{~h}$ of remodelling by the fibroblasts on a domain of $0.5 \mathrm{~mm}$ by $1.0 \mathrm{~mm}$. The cells have a speed of $15 \mu \mathrm{m} \mathrm{h}^{-1}, \rho=0, k=0.15 \mathrm{~h}$ and the numerical grid for the vector field is 101 by 51 .

In this extension, the matrix is still modelled as a vector field but the vectors are no longer unit vectors. The length of the vector represents the density of the fibrous protein at that location and we model both collagen and fibrin.

The model has the same framework as before and the same interactions of contact guidance and cells orienting the fibrous matrix. In this model, the matrix is composed of two vectors $\boldsymbol{c}$ and $\boldsymbol{b}$ for the collagen and the fibrin, respectively. The length of the vectors represents the density of each protein. The cells produce collagen at a constant rate and degrade collagen at a rate proportional to the amount of collagen already present. They also degrade fibrin at a rate proportional to the amount present. In addition, the cell speed is regulated by the density of the proteins. The speed function is taken to be the product of a linearly increasing function of fibrin density (we assume that fibronectin is proportional to the density of fibrin) and a decreasing function of collagen density.

The new model is described by the following equations:

$$
\begin{aligned}
& \boldsymbol{u}^{i}(t)=(1-\alpha) \boldsymbol{c}\left(\boldsymbol{f}^{i}(t), t\right)+\alpha \boldsymbol{b}\left(\boldsymbol{f}^{i}(t), t\right), \\
& \boldsymbol{v}^{i}(t)=(1-\rho) \frac{\boldsymbol{u}^{i}(t)}{\left\|\boldsymbol{u}^{i}(t)\right\|}+\rho \frac{\dot{\boldsymbol{f}}^{i}(t-\tau)}{\left\|\dot{\boldsymbol{f}}^{i}(t-\tau)\right\|},
\end{aligned}
$$

which allows the cells to be polarized (parametrized by $\rho$ ), a property we discuss later. The equation determining $\boldsymbol{f}^{i}$ is given by

$$
\dot{\boldsymbol{f}}^{i}(t)=s\left(\left\|\boldsymbol{c}\left(\boldsymbol{f}^{i}(t), t\right)\right\|,\left\|\boldsymbol{b}\left(\boldsymbol{f}^{i}(t), t\right)\right\|\right) \frac{\boldsymbol{v}^{i}(t)}{\left\|\boldsymbol{v}^{i}(t)\right\|},
$$




$$
\begin{gathered}
\boldsymbol{f}(\boldsymbol{x}, t)=\sum_{i=0}^{M} w\left(\boldsymbol{f}^{i}(t)-\boldsymbol{x}\right) \frac{\dot{\boldsymbol{f}}^{i}(t-\tau)}{\left\|\dot{\boldsymbol{f}}^{i}(t-\tau)\right\|}, \\
\frac{\mathrm{d} \theta(t)}{\mathrm{d} t}=\kappa\|\boldsymbol{f}\| \sin (\phi-\theta),
\end{gathered}
$$

where $\theta$ is the direction of the collagen matrix at a point in space, $\kappa$ is a parameter which measures the ability of the fibroblasts to reorder the matrix and $\phi$ is the direction of the vector $f$. Hence

$$
\begin{gathered}
\frac{\mathrm{d}\|\boldsymbol{c}\|}{\mathrm{d} t}=\left(p_{c}-d_{c}\|\boldsymbol{c}\|\right) \sum_{i=1}^{M} w\left(\boldsymbol{f}^{i}-\boldsymbol{x}\right), \\
\frac{\mathrm{d}\|\boldsymbol{b}\|}{\mathrm{d} t}=-d_{b}\|\boldsymbol{b}\| \sum_{i=1}^{M} w\left(\boldsymbol{f}^{i}-\boldsymbol{x}\right),
\end{gathered}
$$

where $p_{c}, d_{c}$ and $d_{b}$ are positive constants.

Simulations from this model indicate that changes in the production and degradation rates of the proteins affect the alignment in an indirect manner. By changing these rates, the densities of the proteins are altered. This affects the speed of the cells, which, as our previous model has already indicated, significantly influences the alignment pattern.

Examining transitions from a collagen-rich environment to one rich in fibrin gives some additional insight, since cells interact differently with each of these proteins. Figure 2 shows that alignment in the collagen-rich region is significantly greater than that observed in the initially fibrin-rich region. In addition, a layer of collagen oriented parallel to the initial protein boundary is evident. A simulation using constant cell speed exhibited similar effects.

The final orientation of the matrix is explained as follows. There are two ways in which the fibroblasts can interact with the fibrin and the collagen. Firstly, the cells reorient collagen but not fibrin, and secondly the cells move faster in fibrin. The fixed speed simulation (figure $2 b$ ) indicates that the difference in matrix orientation is not due to speed changes, but is due to the way in which cells reorient the fibres. The fact that cells do not alter fibrin orientation means that its initial orientation (which was random in these simulations) will continue to affect the direction of the cells until it is totally degraded. This has a randomizing effect and the final orientation of the matrix is more random in the region that was initially fibrin-rich. The boundary layer is also a result of the randomizing influence of the fibrin, as the cells are more likely to turn when they enter the fibrin.

\section{Modelling transforming growth factor- $\beta$ in dermal wound repair}

In this section, we describe an extension of the model designed to explore the effects of transforming growth factor- $\beta$ (TGF $\beta$ ) on wound healing (Dallon et al. 2001). It has been demonstrated that either neutralizing TGF $\beta_{1}$ and $\mathrm{TGF} \beta_{2}$ or adding $\mathrm{TGF} \beta_{3}$ will reduce the extent of scarring in a wound. TGF $\beta$ alters the wound healing process in many ways however (Roberts \& Sporn 1996; O'Kane 

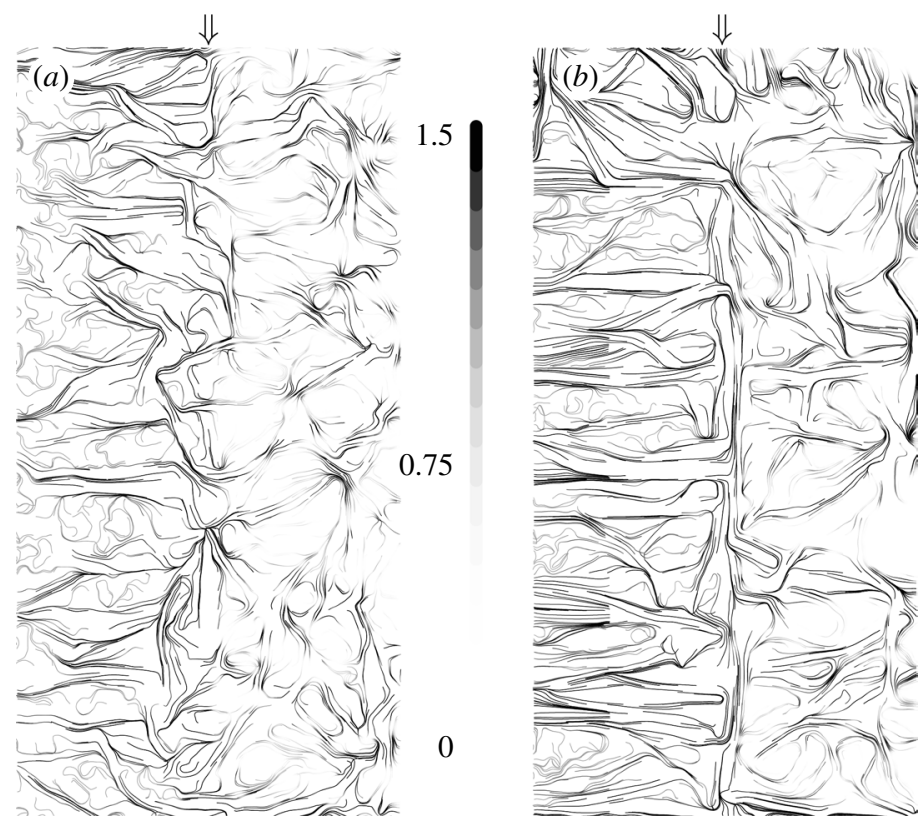

Figure 2. Alignment patterns showing the effect of an initial transition from collagen to fibrin. The alignment is stronger on the left side of the domain where the initial protein was collagen and weaker on the right side where the initial protein was fibrin. The position of the initial interface between collagen and fibrin is indicated by the arrow. In $(a)$, the cell speed depends on the protein densities with an average speed of $3.11 \mu \mathrm{m} \mathrm{h}^{-1}, s=15$ and the total collagen density is 1.14. In $(b)$, the cell speed is constant and set at $5 \mu \mathrm{m} \mathrm{h}^{-1}$. Three hundred cells are initially placed along the collagen fibrin interface with randomly chosen directions.

\& Ferguson 1997), and it is unclear which precise effects are responsible for changing the degree of scarring. It is known that TGF $\beta$ has various effects on fibroblasts: it acts as a chemoattractant, modulates collagen production and degradation as well as cell proliferation (Clark et al. 1995; Cordeiro et al. 2000). Most experimental evidence suggests that the three isoforms affect fibroblasts similarly (Locci et al. 1999; Cordeiro et al. 2000). Two studies indicate some differences however; one in the way the collagen production is altered (Murata et al. 1997) and the other in the formation of appendages in mesenchymal cells (Taya et al. 1999). The second study suggests that TGF $\beta_{3}$ increases the production of filopodia, whereas TGF $\beta_{1}$ and $\mathrm{TGF} \beta_{2}$ promote the formation of lamellipodia.

In order to determine which of the effects of TGF $\beta$ are important in explaining how this cytokine affects scar formation, we allow several parameters in our model to vary with the concentration of TGF $\beta$. The equations for the model are the same as equations (4.1)-(4.7) with the following changes. The speed function in equation (4.3) is now a function of collagen density, fibrin density and TGF $\beta$ concentration, $s\left(\left\|\boldsymbol{c}\left(\boldsymbol{f}^{i}(t), t\right)\right\|,\left\|\boldsymbol{b}\left(\boldsymbol{f}^{i}(t), t\right)\right\|\right.$, TGF $\left.\beta\right)$. The constant parameters $p_{c}$, $d_{c}$ and $d_{b}$ in equations (4.6) and (4.7) are now all functions of TGF $\beta$ concentration. Finally, in this model, the cells are allowed to proliferate and the rate at which they proliferate depends upon the TGF $\beta$ concentration. Cell proliferation is modelled by introducing two populations of fibroblasts: those which divide 
and those which do not. Dividing fibroblasts become non-dividing fibroblasts with time. Each cell has a clock, not synchronized with the other cells, which determines when it should divide. When a cell divides it loses its polarity and the daughter cell is placed at a small distance $(10 \mu \mathrm{m})$ from the mother cell.

Experimental evidence shows that the concentration of active TGF $\beta$ peaks within an hour after wounding, decreases to a level above normal within 1 day, peaks again 5 days after wounding and then decreases more gradually until reaching approximately normal levels 14 days after wounding. Since fibroblasts do not enter the wound until $24-48 \mathrm{~h}$ after wounding, we ignore the first peak in TGF $\beta$ concentration and assume it to be a function of time as described earlier.

Simulations from this model suggest that the effect of TGF $\beta$ on fibroblast speed, proliferation and collagen production cannot explain its effect on scar tissue formation (see figure $3 a, b$ ). However, the simulations suggest that the effect on scarring could be explained if TGF $\beta_{3}$ causes a cell to change direction more frequently than TGF $\beta_{1}$ and TGF $\beta_{2}$. For example, if TGF $\beta_{3}$ causes a cell to be more responsive to matrix cues in determining its direction than TGF $\beta_{1}$ and TGF $\beta_{2}$, and if the three isoforms of TGF $\beta$ bind competitively, our simulations give an explanation of the anti-scarring properties of $T G F \beta_{3}$ which is consistent with the experimental data.

\section{Extending the model to include a diffusible chemoattractant}

The above models of Dallon et al. consider the wound space as an isolated region, with fibroblasts entering through the base and the sides in a predetermined way. In reality, the wound resides within intact, uninjured skin, and the fibroblasts enter the wound by migrating from this surrounding tissue. It is relatively straightforward to extend the model to include a band of normal tissue around the wound. One simply makes the computational domain larger, and specifies an initial region as wounded (fibrin-based ECM and no fibroblasts), with the remaining tissue unwounded (collagen-based ECM, with a resident fibroblast population). However, numerical solutions of the model on such an enlarged domain do not predict healing on a reasonable time-scale (figure 4). The movement of fibroblasts into the wound region is extremely slow-many times slower than the rates imposed by Dallon et al. As a result, the repair process occurs at a rate many times slower than that observed in practice.

The slow progress of repair in simulations such as that shown in figure 4 occurs because fibroblasts only enter the wound region as part of their intrinsic movement in normal tissue unbiased by chemoattractants present in wound healing. In reality, fibroblast movement is actively directed towards the wound during healing by a range of chemical cues. For example, PDGF, IL-1 $\beta$ and TNF$\alpha$ are all produced by white blood cells during the inflammatory phase of healing, and are established chemoattractants for fibroblasts (Kim et al. 1999; Sasaki et al. 2000).

To incorporate this directed movement into our model, we assume that a single generic chemoattractant is produced in the wound and diffuses into surrounding tissue; for simplicity, we assume the production rate to be uniform in the wound, with a decay rate that is the same in the wound and in the surrounding tissue. This gives a simple equation for the chemoattractant 


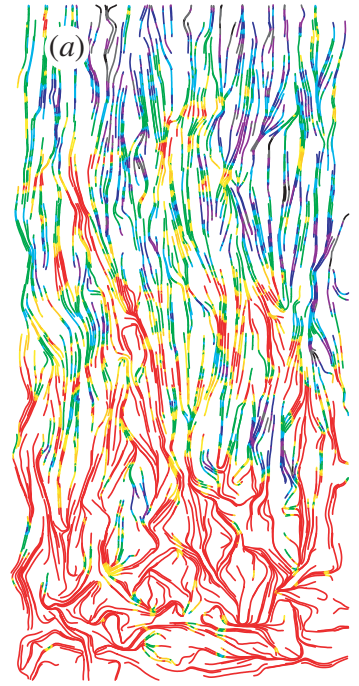

normal

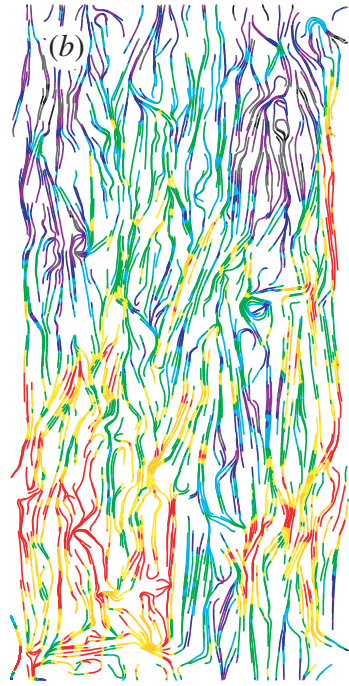

no TGF $\beta$

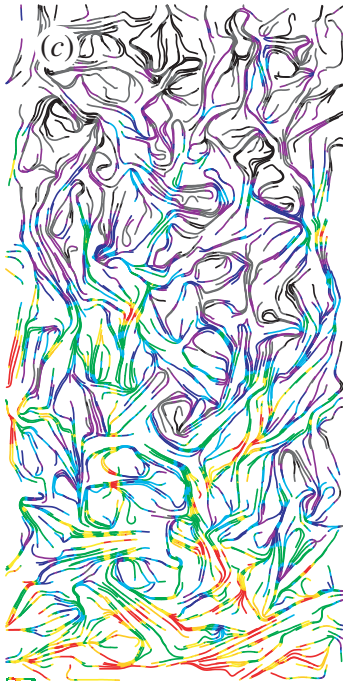

anti-TGF $\beta_{1}$

Figure 3. The collagen matrix for a simulation of normal wound healing with TGF $\beta$, resulting in a scar, is shown in $(a)$. In $(b)$, we show a simulation of a wound treated with an antibody for TGF $\beta$ assuming TGF $\beta$ alters cell proliferation, cell motility and flux, and collagen production. The simulation suggests that these effects do not explain the effects on scar tissue formation of TGF $\beta$. In $(c)$, we show the results for a numerical experiment simulating anti-TGF $\beta_{1}$ treatment, showing a more disordered collagen fibre pattern that is highly reminiscent of experimental data. The more disordered matrix pattern is a key component of reduced scarring. The parameters for this simulation are identical to $(b)$, except that the cells reorient approximately every 9 min instead of every $27 \mathrm{~min}$. The ability of a cell to change direction depends on the time discretization used when integrating equation (4.3). The collagen matrix is represented after $240 \mathrm{~h}$ of simulation corresponding to about 12 days after wounding (the fibroblasts enter the wound region between 24 and $48 \mathrm{~h}$ after wounding). Red indicates high density and dark blue indicates low collagen. The line segments are streamlines for the collagen vector field. The region represents a cross-section of a slash wound with the surface of the skin at the top, normal tissue to the right and left and the basement membrane at the bottom. When combining the three effects of TGF $\beta$, we assume that the cell proliferation increases at $90 \mathrm{pg} \mathrm{mm}^{-3}$ of TGF $\beta$ and then decreases for higher concentrations; for the motility we combine the decreased cell speed and the elevated flux at $90 \mathrm{pg} \mathrm{mm^{-3 }}$ of TGF $\beta$ which decreases with increased concentrations of TGF $\beta$.

concentration $A(\boldsymbol{x}, t)$ of the form

$$
\partial A / \partial t=D_{A} \nabla^{2} A-k_{1} A+ \begin{cases}k_{2} & \text { in the wound, } \\ 0 & \text { in the surrounding tissue. }\end{cases}
$$

Chemoattractant production begins during the inflammation phase of repair, several days earlier than the fibroblast activity at the centre of our model. Within this period, equation (6.1) effectively reaches an equilibrium, and we assume that the chemoattractant profile remains at this equilibrium throughout the dermal healing phase. A typical chemical profile is illustrated in figure 5 .

We modify the cell movement rules to take account of this chemoattractant. We assume that both the speed and direction of the cell vary with the gradient 


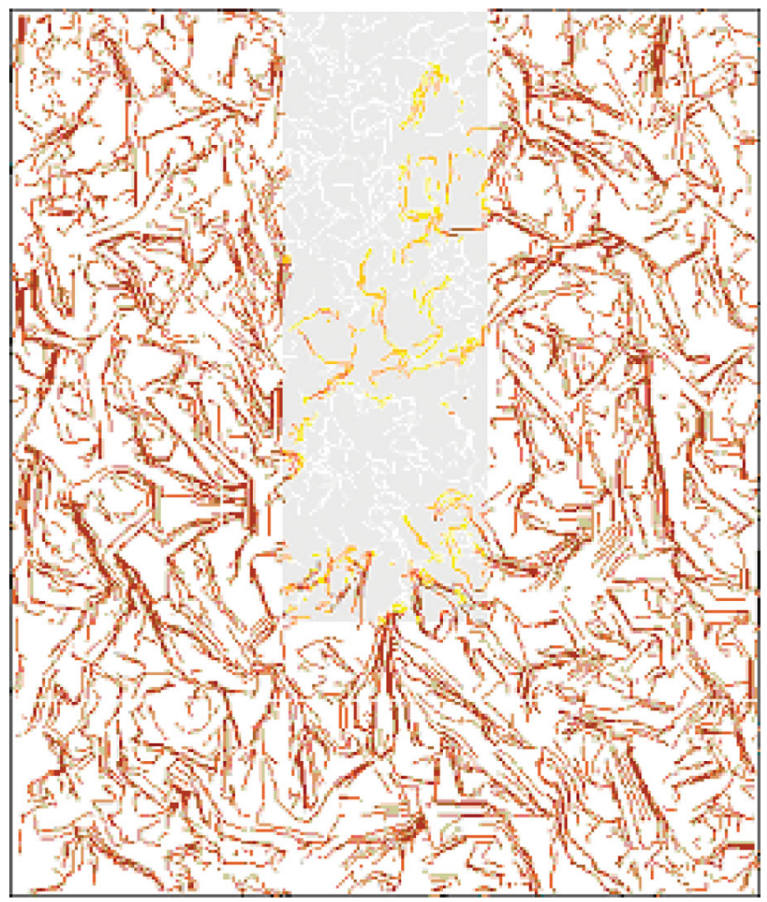

Figure 4. Solution of the model of Dallon et al. on an enlarged domain, which includes a region of unwounded tissue around the wound. The solution shown is after $100 \mathrm{~h}$ of the proliferative phase of healing, which corresponds to about 7 days post-wounding. In the simulation, very little healing has occurred, whereas in reality the wound would be significantly repaired by this time. These results indicate that the model must be amended to include the directed movement of fibroblasts into the wound. The model details and parameter values are as in $\S 4$, with $\alpha=0.5, \rho=0.5$, $s=15 \mu \mathrm{m} \mathrm{h}^{-1}, \kappa=5, p_{c}=0.64, d_{c}=0.44, d_{b}=0.6$ and $\tau=0.15 \mathrm{~h}$. Eight hundred fibroblasts are initially resident in the unwounded dermis, and mitosis occurs on average every $18 \mathrm{~h}$.

$\nabla A$ of the chemoattractant. For the cell speed, we alter the form to

$$
s_{\text {new }}=s_{\text {old }}(\|\boldsymbol{c}\|,\|\boldsymbol{b}\|)\left(1+\|\nabla A\| /\|\nabla A\|_{\max }\right) .
$$

Here, $s_{\text {old }}$ is the speed assumed in the previous model, given in equation (4.3), and $\|\nabla A\|_{\max }$ is the maximum value of $\|\nabla A\|$ across the domain. Thus, cell speed is an increasing function of chemoattractant gradient. We assume that cell direction is modified by the chemical gradient in a manner that is directly analogous to the affects of collagen and fibrin densities; thus the cell direction is given by

$$
\boldsymbol{v}_{\text {new }}=\frac{\left(1-\rho_{2}\right) \boldsymbol{v}_{\text {old }}+\rho_{2} \nabla A /\|\nabla A\|}{\left\|\left[\left(1-\rho_{2}\right) \boldsymbol{v}_{\text {old }}+\rho_{2} \nabla A /\|\nabla A\|\right]\right\|} .
$$

Here, the unit vector $\boldsymbol{v}_{\text {old }}$ is the cell direction assumed in the previous model, given in equation (4.2). The quantity $\rho_{2}$ reflects the dependence of cell direction on chemoattractant gradient, and we assume that it is an increasing, saturating function of this gradient, with a Hill function form

$$
\rho_{2}=\|\nabla A\|^{\alpha} /\left[\|\nabla A\|_{\text {crit }}^{\alpha}+\|\nabla A\|^{\alpha}\right] .
$$

Here, $\alpha$ and $\|\nabla A\|_{\text {crit }}$ are positive parameters. 


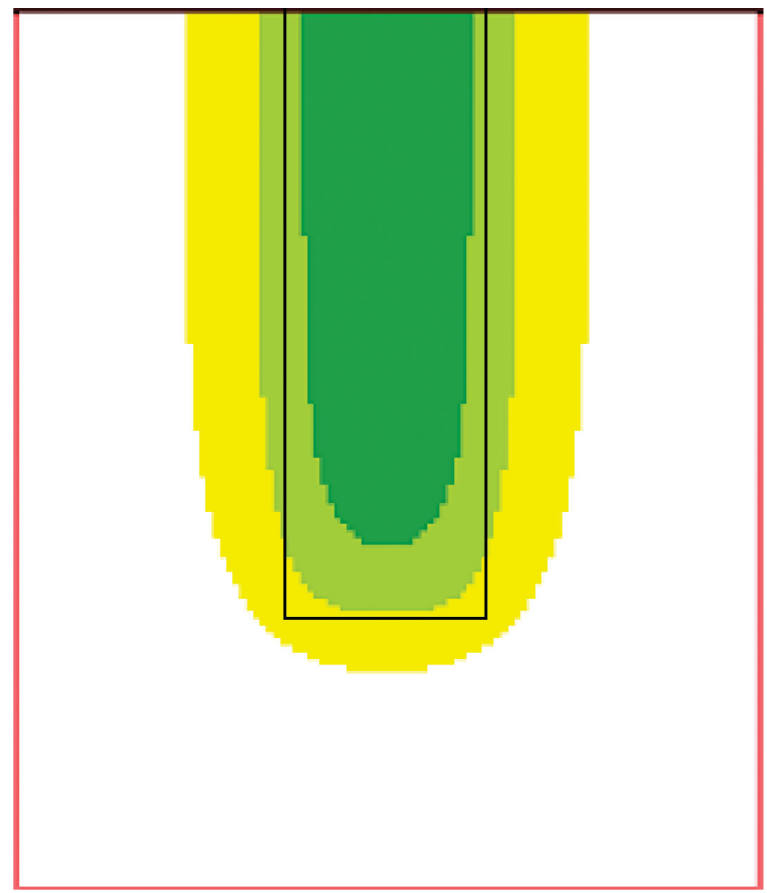

Figure 5. A typical chemoattractant profile, as predicted by equation (6.1). The chemoattractant concentration is greatest in the wound, and gradually decreases in the wound periphery. The resultant chemical gradient directs fibroblasts into the wound region. The parameter values are $D_{A}=0.5, k_{1}=2.0 \times 10^{-4}, k_{2}=0.025$.

One final change to the model is required. Dallon et al. assume that the majority of the fibroblasts enter the wound through its base, with only a minority entering through the sides. This assumption is based on cell staining experiments of Adams (1997), and reflects the significant increase in fibroblast density with depth in unwounded dermis. Thus, the upper part of the dermis, near the epidermis, is relatively acellular, whereas the part near the fascia has a high density of fibroblasts. In the model, we simply impose a linear variation in fibroblast density as an initial condition; the limited fibroblast movement within normal tissue means that the density gradient is maintained over the time-scale of wound repair. The initial linear density gradient is achieved by inverting a linear probability distribution function of fibroblast density as a function of depth from the skin surface. Assuming no fibroblasts at the skin surface leads to the following equation for determining the vertical coordinate $\left(y_{\mathrm{FB}}\right)$ of a given fibroblast: $y_{\mathrm{FB}}=y_{\max }(1-\sqrt{1-\mathrm{rnd}})$ where $y_{\max }$ corresponds to the base of the simulation domain and rnd is a random number between 0 and 1 .

Figure 6 shows a typical solution of the extended model. We plot the collagen profile both during the early stages of repair and after $100 \mathrm{~h}$ of the proliferative phase of healing (this corresponds to about 7 days post-wounding). A steady gradient of a chemoattractant is rapidly established, and this directs fibroblasts into the wound, so that healing proceeds at a similar rate to that observed in practice. As in the previous model, the fibroblasts break down the initial fibrinbased matrix and replace it with collagen. Initially, collagen fibres are laid down 

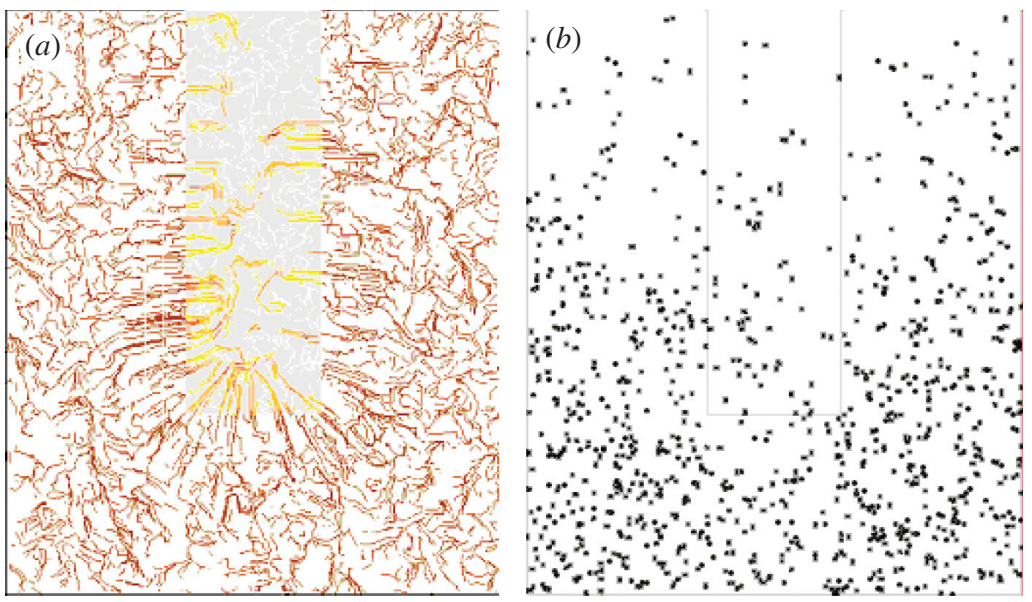

(c)
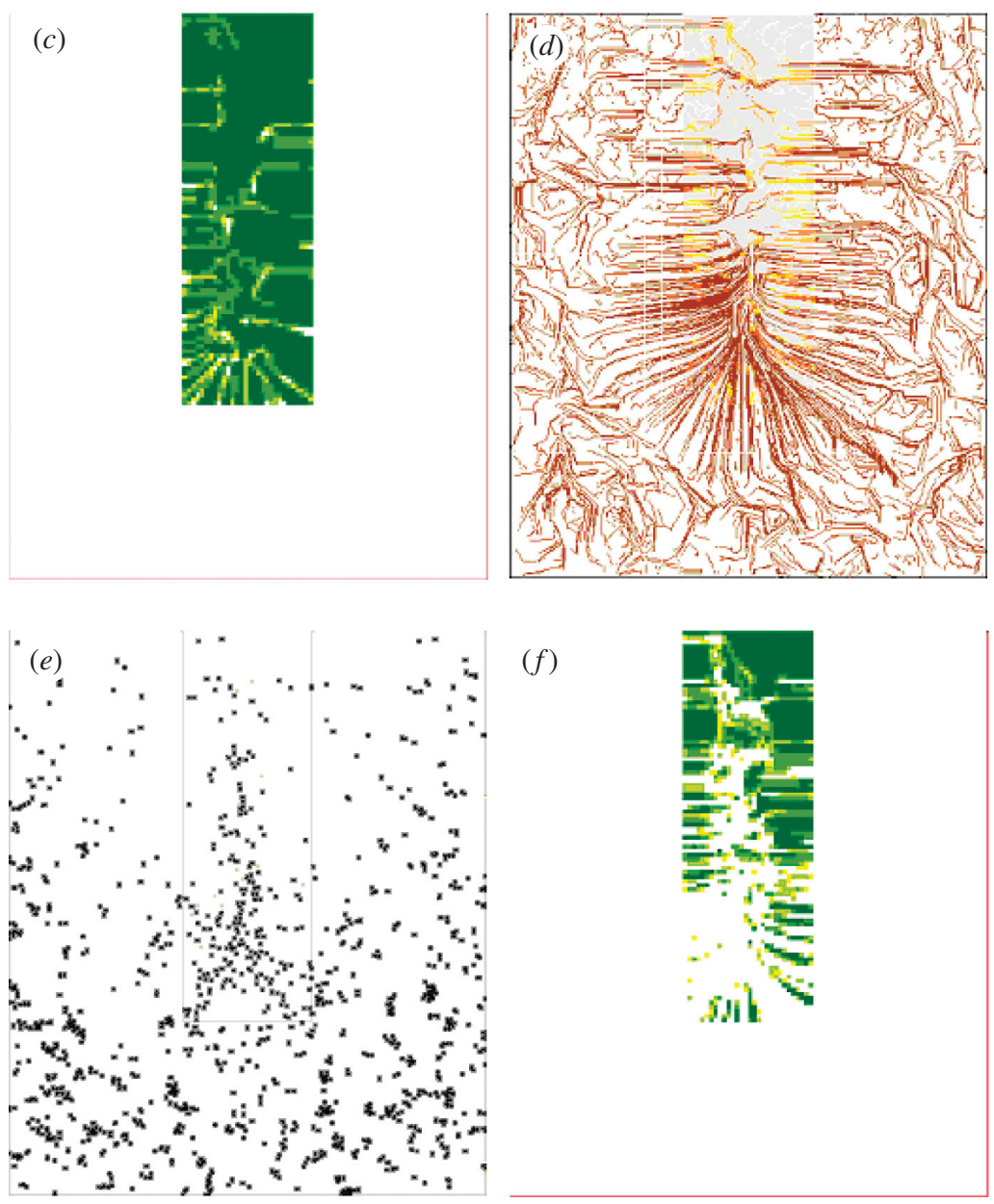

$(f)$

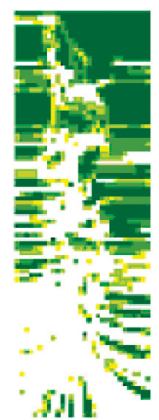

Figure 6. (Caption overleaf.) 
Figure 6. (Overleaf.) A typical simulation of the extended model, showing wound repair orchestrated by a chemoattractant for fibroblasts, produced within the wound space. We show the collagen $(a, d)$, fibroblast $(b, e)$ and fibrin $(c, f)$ profiles at $(a-c) 25$ and $(d-f) 100 \mathrm{~h}$ after the start of the proliferative phase of healing (which itself begins about 3 days after injury). The fibroblasts move rapidly into the wound in response to the chemical gradient, break down the fibrin matrix, and replace it with collagen fibres whose alignment reflects the direction of fibroblast migration. The parameter values are $D_{A}=0.5, k_{1}=2.0 \times 10^{-4}, k_{2}=0.025,\|\nabla A\|_{\text {crit }}=0.5\|\nabla A\|_{\max }, \alpha=10$. Other model details and parameter values are as in figure 3 .
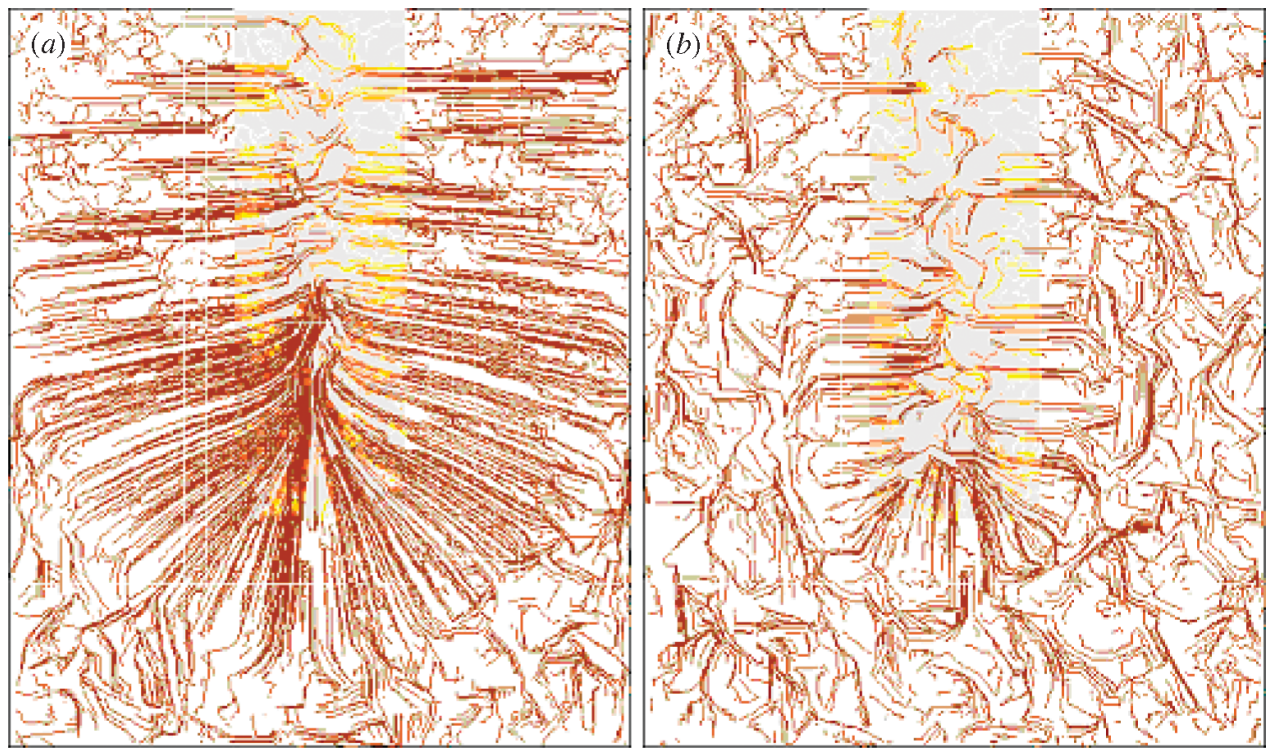

Figure 7. An illustration of the change in collagen profile with the steepness of the chemoattractant gradient. (a) A highly diffuse chemoattractant causes fibroblasts to move towards the wound, and thus reorient the collagen matrix, over a large region of unwounded tissue. (b) Conversely, a highly localized chemoattractant only affects the movement of fibroblasts close to the wound periphery. Therefore, in $(a)$ the wound and normal tissue matrices are highly interwoven, whereas in $(b)$, they are relatively distinct, resulting in a much greater risk of rupture. The parameter values are as in figure 6 except for $(a) D_{A}=2.0$ and $(b) D_{A}=0.1$.

in the direction of fibroblast movement, and this orientation pattern is reinforced by the tendency of successive fibroblasts to move along the existing fibres. In this extended model, fibroblast direction is also strongly influenced by the chemoattractant profile, and as a result the collagen fibre orientation pattern reflects the gradient of chemoattractant. In figure 6, we also show the fibroblast distribution. This illustrates the gradient of fibroblast density in unwounded skin, and shows that this is reflected in the wound, with significantly higher cell numbers at the base of the wound compared to the top.

Our extended model demonstrates that the rapid influx of fibroblasts into the wound space can be explained simply as a result of a chemoattractant gradient, coupled with the variation of fibroblast density with depth in unwounded dermis. The new model also enables the study of an important feature of wound repair that is outside the scope of the model of Dallon et al., namely the extent to which the collagen matrix around the wound is altered during healing. In figure 6 , there 

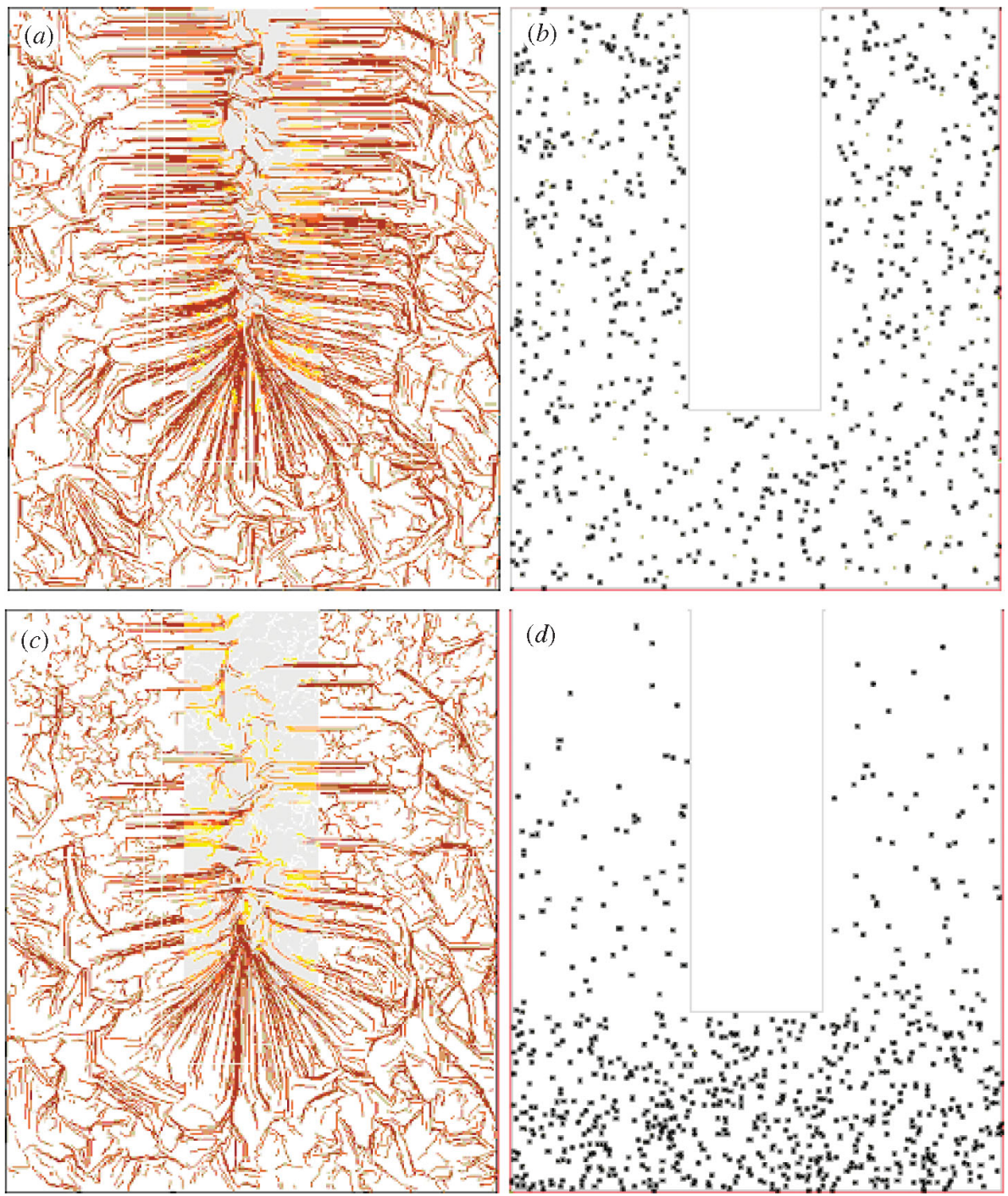

Figure 8. An illustration of the dependence of wound collagen profile on the variation of fibroblast density with depth in unwounded dermis. In $(a)$, we show the effect of assuming uniform fibroblast density in the dermis: there is a much more uniform deposition of collagen than in the reference solution (figure 6). In (b), we use a fibroblast density that is heavily skewed towards the base of the dermis; in this case the collagen fibres in the wound are predominantly oriented perpendicular to the skin surface because most fibroblasts enter the wound through its base. The model details and parameter values are as in figure 6 , except for the fibroblast distribution. The total number of dermal fibroblasts is the same in $(a),(b)$ and in figure 6 ; in $(b)$, the distribution of fibroblasts has $20 \%$ of fibroblasts above the wound base, distributed as a linear function of depth in the dermis, and $80 \%$ below the wound base.

is a clear reorientation of the collagen fibres around the wound, over a distance of about half the wound width $(0.25 \mathrm{~mm})$. This is caused by the fibroblasts remodelling the matrix as they move towards the wound. This reorientation of the existing collagen matrix results in an interdigitation of collagen fibres in 


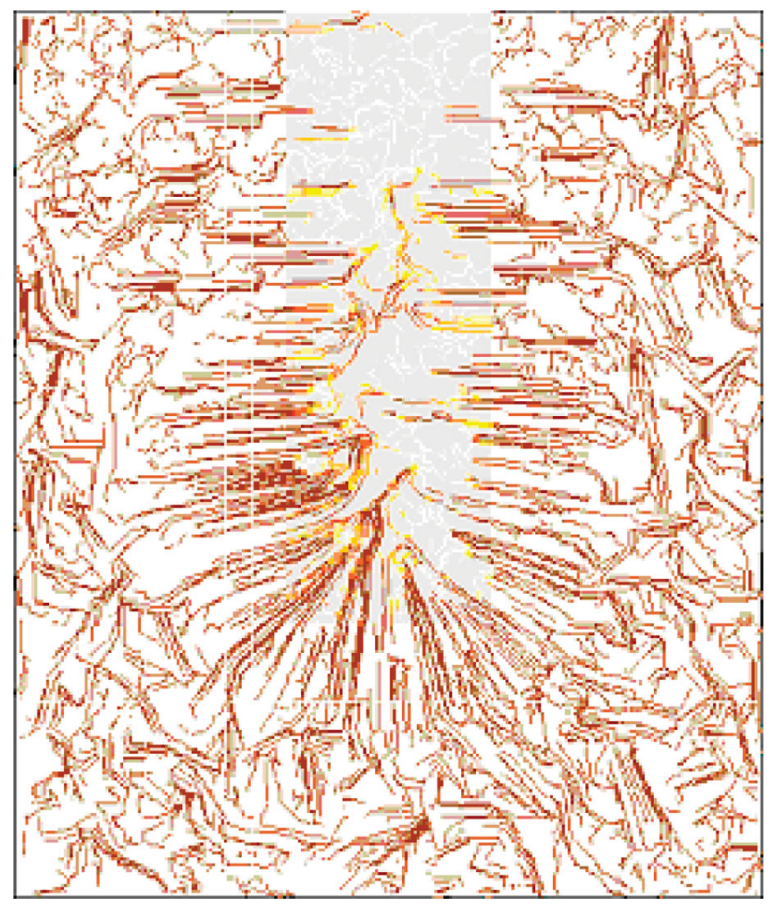

Figure 9. The effects of neglecting the dependence of fibroblast speed on chemoattractant gradient. Fewer fibroblasts enter the wound in comparison to the reference case (figure 6), and as a result the rate of collagen deposition is lower, but the orientation pattern remains broadly similar. The model details and parameter values are as in figure 6 except that the cell speed is given by $s_{\text {old }}(\|\boldsymbol{c}\|,\|\boldsymbol{b}\|)$.

the scar and the surrounding tissue, which is a crucial factor for wound strength. By varying the parameters associated with the chemoattractant in our model, we have found that the degree of interdigitation depends critically on the spread of chemoattractant into the tissue around the wound. A very widely dispersed chemoattractant profile leads to fibroblast recruitment into the wound from a large area of surrounding tissue, and a consequently high degree of interdigitation (figure 7). Similarly, a highly localized chemoattractant profile causes the scar and the surrounding tissue to be poorly linked (figure 7). Therefore, our model makes the novel prediction that wound strength could be improved by manipulation of the chemical gradients set up during the inflammatory phase of repair. Moreover, experimental measurements on the extent of interdigitation could be used to better constrain some of our model parameters.

\section{(a) The role of fibroblast distribution}

In figure 6 , we have imposed a variation in fibroblast density with unwounded dermis, with higher cell densities at greater depths. Such a variation occurs in practice, but we are not aware of any quantitative data from which an appropriate functional form could be estimated. This makes it important to study the way in which the healing profile changes with the assumed fibroblast distribution. In figure $8 a$, we show the effects of neglecting the cell density 


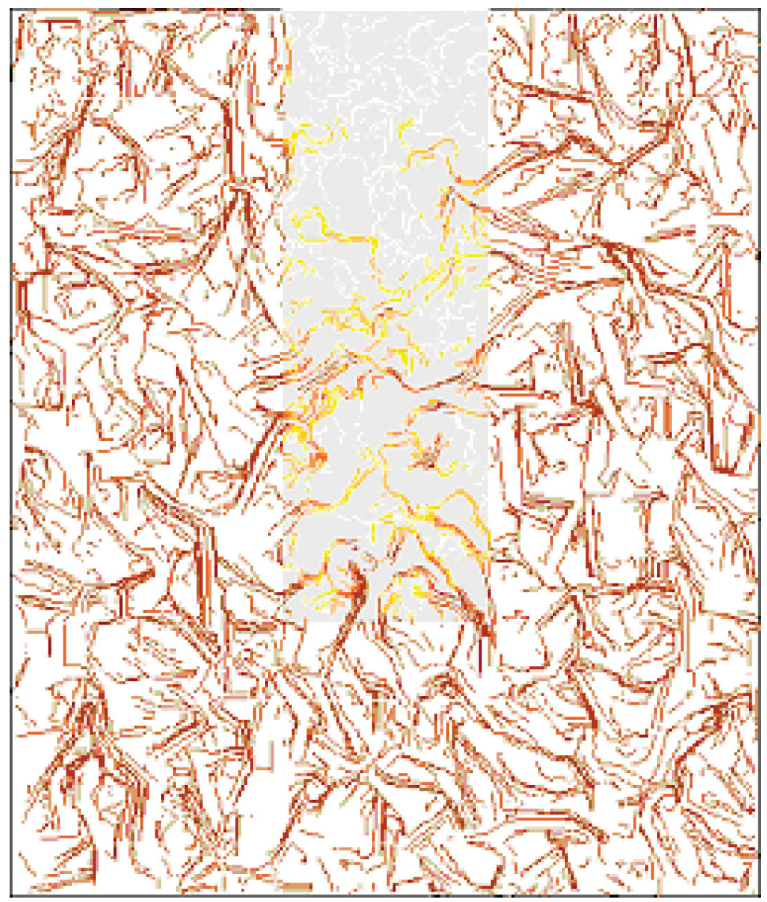

Figure 10. The effects of neglecting the dependence of fibroblast direction on chemoattractant gradient. Significantly fewer fibroblasts enter the wound in comparison to the reference case (figure 6), and as a result the rate of collagen deposition is much lower. In addition, the orientation pattern is significantly altered, with a much more disordered appearance that is more reminiscent of unwounded dermis. The model details and parameter values are as in figure 6 except that the cell direction is given by $\boldsymbol{v}_{\text {old }}$.

gradient and assuming a uniform distribution of fibroblasts in the dermis. In comparison to the reference solution in figure 6 , there is a much more uniform deposition of collagen in the wound, and a higher degree of fibre alignment parallel to the skin surface. This latter effect is a result of more cells entering the wound through the sides rather than the base. This alignment is greatly reduced if we assume that the fibroblast distribution is heavily skewed towards the lower parts of the dermis (figure $8 b$ ). In this case, most of the cells entering the wound do so through the base, simply because of the high cell number below the wound, and as a result the wound collagen at later times has a predominant orientation perpendicular to the skin surface.

\section{(b) The activity of the chemoattractant}

The chemoattractant plays a key role in orchestrating fibroblast movement into the wound. In our model, we assume that both the speed and direction of cell movement are regulated by the chemoattractant gradient, and it is important to understand how these two separate effects conspire to produce the observed healing pattern. In figure 9, we show the collagen pattern given by neglecting the dependence of cell speed on chemoattractant gradient, so that only cell direction is 


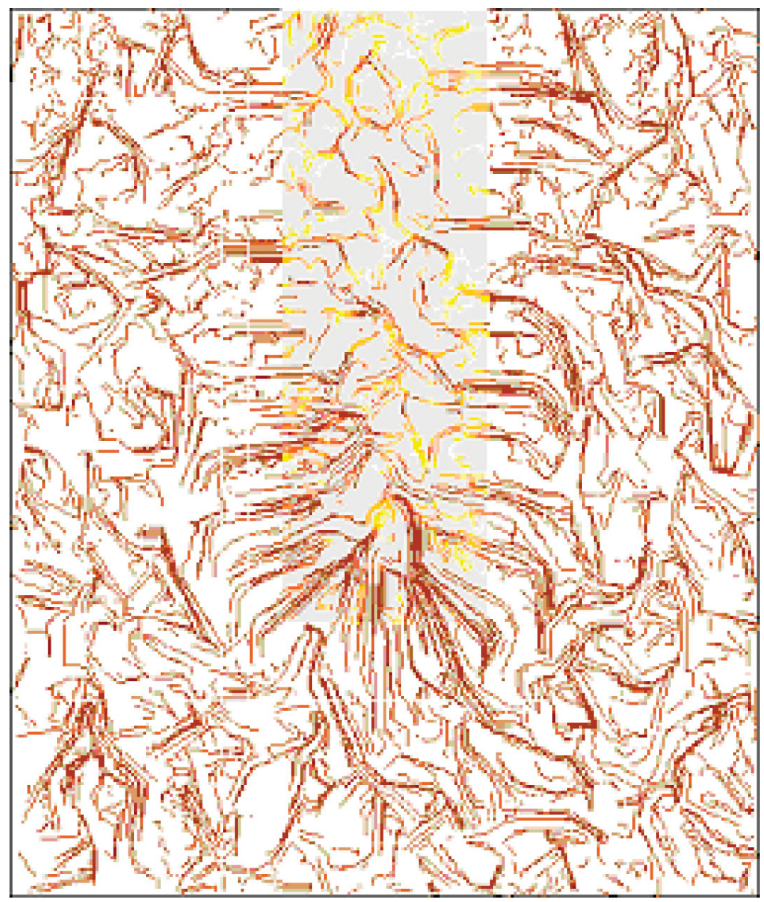

Figure 11. The effects of changing the way in which the chemoattractant gradient alters cell direction. This simulation is a crude representation of the addition of an agent that binds competitively to the chemoattractant receptors on the fibroblast surface. The collagen fibres in the wound are much more disordered than in the reference case (figure 6), corresponding to higher scar quality. The model details and parameter values are as in figure 6 , except that $\|\nabla A\|_{\text {crit }}=$ $0.9\|\nabla A\|_{\max }$ and $\alpha=10$.

affected by the chemical. As expected, fewer fibroblasts enter the wound space than in the reference case (figure 6), and as a result the rate of collagen deposition is lower, but the orientation pattern of the collagen fibres is not significantly altered. Neglecting the dependence of cell direction (but not speed) on chemoattractant reduces the fibroblast influx into the wound even further, and hence the collagen deposition is markedly reduced (figure 10). But in addition, the orientation pattern of the collagen fibres is significantly altered, with a more random distribution that is much closer to unwounded dermis.

The latter result suggests that the degree of scarring may depend critically on the way in which the cell orientation is regulated by chemoattractant gradient - mathematically, this means the dependence of $\rho_{2}$ on $\nabla A$, which is an increasing saturating function, and which we represent mathematically as a Hill function. In a series of 'mathematical experiments', we have found that it is possible to significantly alter the orientation pattern of the collagen fibres in the scar by altering the parameters of this Hill function. A gradual increase in cell orientation with chemoattractant gradient results in a highly oriented collagen matrix, both within the scar and in the surrounding tissue (not illustrated for brevity). This is because the cells reorient and move towards the wound over a large region of intact dermis, and continue this directed movement throughout 
the wound. Conversely, if the chemical gradient has little effect on the cell orientation when it is small, with a steep increase in the cell orientation response at higher gradients, the collagen pattern in the wound is much more disordered (figure 11), corresponding to high scar quality. In figure 11, the fibroblasts close to the wound edge are directed into the wound by the high chemoattractant gradients at the wound edge. However, the chemical has little effect on fibroblast direction either far from the wound, or within the wound-because the chemical gradient is relatively low at both locations. Once in the wound, fibroblast movement is regulated mainly by ECM composition and orientation, and this results in a disordered scar matrix, such as that shown in figure 11.

\section{Discussion}

We have presented a multiscale modelling framework which allows us to analyse the effects of different factors on collagen alignment and scar formation during dermal wound healing. From a therapeutic point of view, these results suggest an anti-scarring treatment that decreases the sensitivity of fibroblast reorientation to chemoattractant gradients. This could be achieved in practice by adding an agent that binds preferentially to the chemoattractant receptors on the cell surface. The anti-scarring agent mannose-6-phosphate acts via exactly this type of competitive inhibition, binding preferentially to TGF $\beta$ receptors, thereby reducing the effective concentration of TGF $\beta$ (Ferguson \& O'Kane 2004). It has been proposed that the lower degree of scarring that results from this treatment is due to changes in fibroblast motility (Taya et al. 1999; Dallon et al. 2001). Our results suggest that in fact, changes in effective chemotactic activity of TGF $\beta$ may also be an important contribution to the anti-scarring effect. Moreover, we suggest that competitive inhibition of the binding of other chemoattractants to dermal fibroblasts may represent a novel alternative or supplementary approach to anti-scarring therapy.

\section{Editors' note}

Please see also related communications in this focussed issue by Byrne et al. (2006) and $\mathrm{Lu}$ et al. (2006).

J.A.S. was supported in part by an Advanced Research Fellowship from EPSRC.

\section{References}

Adams, J. J. 1997 The cell kinetics of murine incisional wound healing. Ph.D. thesis, University of Manchester.

Alberts, B., Johnson, A., Lewis, J., Raff, M., Roberts, K. \& Walter, P. 2002 Cell junctions, cell adhesion, and the extracellular matrix. In Molecular biology of the cell, pp. 978-986, 4th edn. New York: Garland Publishing.

Birk, D. E. \& Trelstad, R. L. 1986 Extracellular compartments in tendon morphogenesis: collagen, fibril, bundle, and macroaggregate formation. J. Cell Biol. 103, 231-240. (doi:10.1083/ jcb.103.1.231) 
Byrne, H. M., Alarcon, T., Owen, M. R., Webb, S. D. \& Maini, P. K. 2006 Modelling aspects of cancer dynamics: a review. Phil. Trans. R. Soc. A 364, 1563-1578. (doi:10.1098/rsta.2006.1786)

Clark, R. A. F. 1989 Wound repair. Curr. Opin. Cell Biol. 1, 1000-1008. (doi:10.1016/09550674(89)90072-0)

Clark, R. A. F. 1996 Wound repair overview and general considerations. In The molecular and cellular biology of wound repair (ed. R. A. F. Clark), pp. 3-50, 2nd edn. New York: Plenum Press.

Clark, P., Connolly, P., Curtis, A. S. G. \& Wilkinson, C. C. W. 1990 Topographical control of cell behaviour. II. Multiple grooved substrata. Development 108, 635-644.

Clark, R. A. F., Nielsen, L. D., Welch, M. P. \& McPherson, J. M. 1995 Collagen matrices attenuate the collagen-synthetic response of cultured fibroblasts to TGF- $\beta$. J. Cell Sci. 108, 1251-1261.

Cordeiro, M. F., Bhattacharya, S. S., Schultz, G. S. \& Khaw, P. T. 2000 Tgf- $\beta 1,-\beta 2$, and - $\beta 3$ in vitro: biphasic effects on tenon's fibroblast contraction, proliferation, and migration. Invest. Ophthalmol. Vis. Sci. 41, 756-763.

Dallon, J. C., Sherratt, J. A. \& Maini, P. K. 1999 Mathematical modelling of extracellular matrix dynamics using discrete cells: fiber orientation and tissue regeneration. J. Theor. Biol. 199, 449-471. (doi:10.1006/jtbi.1999.0971)

Dallon, J. C., Sherratt, J. A., Maini, P. K. \& Ferguson, M. W. J. 2000 Biological implications of a discrete mathematical model for collagen deposition and alignment in dermal wound repair. IMA J. Math. Appl. Med. Biol. 17, 379-393.

Dallon, J. C., Sherratt, J. A. \& Maini, P. K. 2001 Modeling the effects of transforming growth factor- $\beta$ on extracellular matrix alignment in dermal wound repair. Wound Repair Regen. 9, 278-286. (doi:10.1046/j.1524-475X.2001.00278.x)

Doillon, C. J., Dunn, M. G., Bert, R. A. \& Silver, F. H. 1985 Collagen deposition during wound repair. Scanning Electron Microsc. 2, 897-903.

Ehrlich, P. H. \& Krummel, T. M. 1996 Regulation of wound healing from a connective tissue perspective. Wound Repair Regen. 4, 203-210. (doi:10.1046/j.1524-475X.1996.40206.x)

Ferguson, M. W. J. \& O'Kane, S. 2004 Scar-free healing: from embryonic mechanisms to adult therapeutic intervention. Phil. Trans. R. Soc. B 359, 839-850. (doi:10.1098/rstb.2004.1475)

Forrest, L. 1983 Current concepts in soft connective tissue wound healing. Br. J. Surg. 70, 133-140.

Friedl, P., Zänker, K. S. \& Bröcker, E. B. 1998 Cell migration strategies in 3-d extracellular matrix: differences in morphology, cell matrix interactions, and integrin function. Microsc. Res. Tech. 43, 369-378. (doi:10.1002/(SICI)1097-0029(19981201)43:5<369::AID-JEMT3 > 3.0.CO;2-6)

Guido, S. \& Tranquillo, R. T. 1993 A methodology for the systematic and quantitative study of cell contact guidance in oriented collagen gels. J. Cell Sci. 105, 317-331.

Hay, E. D. (ed.) 1991 Cell biology of extacellular matrix, 2nd edn. New York: Plenum Press.

Hsieh, P. \& Chen, L. B. 1983 Behavior of cells seeded on isolated fibronectin matrices. J. Cell Biol. 96, 1208-1217. (doi:10.1083/jcb.96.5.1208)

Jennings, R. W. \& Hunt, T. K. 1992 Overview of postnatal wound healing. In Fetal wound healing (ed. N. S. Adzick \& M. T. Longaker), pp. 25-52. New York: Elsevier.

Kim, W., Mohan, R., Mohan, R. \& Wilson, S. 1999 Effect of pdgf, il-1 alpha, and bmp2/4 on corneal fibroblast chemotaxis: expression of the platelet-derived growth factor system in the cornea. Invest. Ophthalmol. Vis. Sci. 40, 1364-1372.

Locci, P., Baroni, T., Lilli, C., Martinese, D., Marinucci, L., Bellocchio, S., Calvitti, M. \& Becchetti, E. 1999 TGF $\beta$ and TGF $\alpha$, antagonistic effect in vitro on extracellular matrix accumulation by chick skin fibroblasts at two distinct embryonic stages. Int. J. Dev. Biol. 43, $157-165$.

Lu, Y., Parker, K. H. \& Wang, W. 2006 Effects of osmotic pressure in the extracellular matrix on tissue deformation. Phil. Trans. R. Soc. A 364, 1407-1422. (doi:10.1098/rsta.2006.1778)

McCallion, R. L. \& Ferguson, M. W. J. 1996 Fetal wound healing and the development of antiscarring therapies for adult wound healing. In The molecular and cellular biology of wound repair (ed. R. A. F. Clark), pp. 561-600, 2nd edn. New York: Plenum Press. 
Murata, H., Zhou, L., Ochoa, S., Hasan, A., Badiavas, E. \& Falanga, V. 1997 TGF- $\beta 3$ stimulates and regulates collagen synthesis through TGF- $\beta 1$-dependent and independent mechanisms. J. Invest. Dermatol. 108, 258-262. (doi:10.1111/1523-1747.ep12286451)

O'Kane, S. \& Ferguson, M. W. J. 1997 Transforming growth factor $\beta$ s and wound healing. Int. J. Biochem. Cell Biol. 29, 63-78. (doi:10.1016/S1357-2725(96)00120-3)

Roberts, A. B. \& Sporn, M. B. 1996 Transforming growth factor- $\beta$. In The molecular and cellular biology of wound repair (ed. R. A. F. Clark), pp. 275-308, 2nd edn. New York: Plenum Press.

Sasaki, M., Kashima, M., Ito, T., Watanabe, A., Izumiyama, N., Sano, M., Kagaya, M., Shioya, T. \& Miura, M. 2000 Differential regulation of metalloproteinase production, proliferation and chemotaxis of human lung fibroblasts by pdgf, interleukin-1 beta and TNF-alpha. Mediators Inflamm. 9, 155-160. (doi:10.1080/09629350020002895)

Taya, Y., O'Kane, S. \& Ferguson, M. W. J. 1999 Pathogenesis of cleft palate in TGF- $\beta 3$ knockout mice. Development 126, 3869-3879.

Whitby, D. J. \& Ferguson, M. W. J. 1991 The extracellular matrix of lip wounds in fetal, neonatal and adult mice. Development 112, 651-668.

Wojciak-Stothard, B., Denyer, M., Mishra, M. \& Brown, R. A. 1997 Adhesion orientation, and movement of cells cultured on ultrathin fibronectin fibers. In Vitro Cell. Dev. Biol. 33, 110-117.

Xu, J. \& Clark, R. A. F. 1996 Extracellular matrix alters PDGF regulation of fibroblast integrins. J. Cell Biol. 132, 239-249. (doi:10.1083/jcb.132.1.239) 